\title{
under Complexity
}

\author{
Jianjun Yu ${ }^{1}$; Xiaosheng Qin, A.M.ASCE ${ }^{2 *}$; Yee Meng Chiew ${ }^{3}$, M. ASCE; \\ Rui Min ${ }^{4}$; Xiling Shen ${ }^{5}$
}

\begin{abstract}
:
\end{abstract}
A stochastic optimization model for urban drainage design (SODD) was proposed in this study to help analyze the tradeoff between investment and acceptable flood damage in urban drainage designs considering effects of both uncertainty and climate change. The simulation model (i.e. SWMM), driven by designed rainfall either from existing Intensity-Duration-Frequency (IDF) curves or future ones subjected to climate change conditions, was used to simulate flooding scenarios. The generalized uncertainty analysis estimation (GLUE) and Monte Carlo simulation methods were employed to quantify the system reliability which was adopted in the constraints of the optimization model. The results from a case study showed that the deterministic optimization was computationally efficient with no randomness encountered in hydrological simulation, although its solution was hardly reliable in achieving the target for flood mitigation. The stochastic version, on the other hand, could offer richer information on system reliability and help managers make a more robust decision. The results also revealed that the rainfall extremes under the impact of climate change could significantly affect system investment. The proposed method is advantageous in facilitating cost-effective planning towards a risk-based drainage design in light of various complexities.

Keywords: Drainage design, stochastic optimization, uncertainty, climate change

\footnotetext{
${ }^{1}$ Postdoctoral Researcher, Environmental Change Institute, School of Geography and the Environment, University of Oxford, Dyson Perrins Building, South Parks Road, Oxford OX1 3QY, UK.

2* Associate Professor, School of Civil and Environmental Engineering, Nanyang Technological University, 50 Nanyang Avenue, Singapore, 639798 (Corresponding Author). E-mail: xsqin@ntu.edu.sg

${ }_{3}$ Professor, School of Civil and Environmental Engineering, Nanyang Technological University, 50 Nanyang Avenue, Singapore, 639798.

${ }^{4}$ Research Associate, School of Civil and Environmental Engineering, Nanyang Technological University, 50 Nanyang Avenue, Singapore, 639798.

${ }^{5}$ Research Associate, DHI Water \& Environment (S) Pte. Ltd., CleanTech One, Singapore, 637141
} 


\section{INTRODUCTION}

Urban drainage systems are designed to accommodate excessive rainfall and avoid threats of urban flooding. It is a critical infrastructure in a modern city to protect human lives, assets, public health and environment. In the past few decades, many areas around the world have suffered from an increasing tendency of climate-change induced extreme storms, which have challenged the existing and aging drainage systems. Particularly, in developing countries (e.g. Southeast Asian countries), rapid urbanization and demographic growth has added to the vulnerability of urban communities to flood hazards (Hammond et al., 2015). Hence, governments are facing urgent needs to improve the capacity of their urban drainage systems by adopting a variety of measures (e.g. low impact development). This necessitates the development of cost-effective drainage designs to achieve a better tradeoff between investment and flood control goals.

Optimization models have been widely adopted to tackle such tradeoff problems in many water resources and environmental engineering fields. Examples of successful applications were found in air quality control (Qin et al., 2010), water supply and irrigation (Dutta et al., 2016), watershed and flood management (Lund, 2012) and climate change adaptation studies (Ashofteh et al., 2015; Marchi et al., 2016). Recently, the heuristic techniques (e.g. genetic algorithm, GA) have received much attention in seeking optimal solutions for various highly-nonlinear water and environmental studies. In terms of drainage system design, Muleta and Boulos (2007) linked a multi-objective optimization model with the EPA Storm Water Management Model (SWMM) (Gironás et al., 2010) to identify the best combination of conduit sizes, storage volumes and pumping capacities in a sewage collection system to meet the flood control requirement at a 
minimum cost. Haghighi and Bakhshipour (2014) presented an integrated optimization model to determine the layout configuration of a sewage collection network using a loop-by-loop cutting algorithm. Cimorelli et al. (2014) and Cozzolino et al. (2015) employed GA, coupled with a steady and uniform flow hydraulic model, to determine optimal parameters (e.g. nodal excavation depths) in a rural drainage network design. Tao et al. (2014) developed a framework to optimize the size of storm water detention tanks in drainage network designs and $\mathrm{Li}$ et al. (2015) further applied to a real world study case. Sebti et al. (2016) compared the capability of the linear programming, GA and simulated anneal algorithms before implementing the best management practice in a combined sewer system.

The optimal design of urban drainage networks, as reported in the aforementioned studies, has some common considerations. Firstly, the main purpose of optimization is to determine the layout configuration and/or network components (e.g. pipe diameters and detention sizes), and the design parameters are normally decision variables in the optimization model. Secondly, the cost arising from the construction and management of drainage rehabilitation, which is represented by a function of the design parameters, is normally a target to be minimized in the optimization process. Thirdly, there exists a series of system constraints associated with hydraulic principles, technical standards, regional limitations and flood control requirements. In such a context, the hydrological/hydraulic models are generally employed to simulate flood scenarios and provide relevant inputs (e.g. surcharge, peak flows and inundation area) to the optimization models. 
71

72

optimization-model-aided drainage design for seeking cost-effective solutions. However, some challenges still exist. Firstly, a simulated urban hydrological system is often impaired by errors or biases, which are caused by the uncertainties associated with input data, system parameters and model structures (Thorndahl and Willems, 2008). The uncertainties that originate from cost estimation could further complicate the problem. Tackling these uncertainties is important to help obtain reliable design solutions. Previously, very few studies in such an area were reported. Jacobs et al. (1997) proposed a chance constrained optimization model to determine strategies for drainage design where the probability of system failure caused by random storm events was considered. Yazdi et al. (2014) reported a study to consider rainfall uncertainty in a drainage rehabilitation planning problem with the purpose of allowing the objective function of overflow reduction being evaluated at a certain percentage of confidence interval. Duan et al. (2016) incorporated numerous optimization models into stochastic analysis to yield the solution of a detention tank design through ranking. Secondly, the drainage design is often carried out under a designed rainfall based on statistical analysis of historical records. How to incorporate the climate-change effect into the drainage design is of particular interest to improve the capability of the drains to adapt to future changes. Mailhot and Duchesne (2010) suggested that the design criteria of urban drainage infrastructure under climate change should integrate the information on projected extreme rainfall, acceptable level of risk and expected lifetime of infrastructures. Similar studies (Zhou et al., 2013; Sebti et al., 2015; Saraswat et al., 2016) also discussed the sustainable infrastructure design and economic benefit analysis to climate-change induced extreme rainfalls. However, these works rarely considered using optimization models. Lastly, it is desired to develop a generic optimization model that is capable of tackling complex uncertainty appropriate for various development options (e.g. pipes, detention tanks and other 
94 low impact development measures).

96 Considering the above stated challenges, the aim of the current paper is to present a Stochastic

97 Optimization model for urban Drainage Design (SODD) subjected to various complexities. The

98

99

100

101

102

103

104 various scenarios.

105

106

107

108

109

110

111

112

113 various scenarios.

\section{METHODOLOGY}

\subsection{The SODD modeling structure}

Figure 1 illustrates the overall workflow of the proposed model and its individual components. Generally, there are two major steps including model establishment and model execution. Model establishment includes optimization model formulation and other components required by SODD such as hydrological model calibration, uncertainty analysis, historical rainfall analysis and climate model downscaling. Model execution is to run the optimization model with customized parameters defined by users to yield the optimal solutions for post-optimization analysis under 


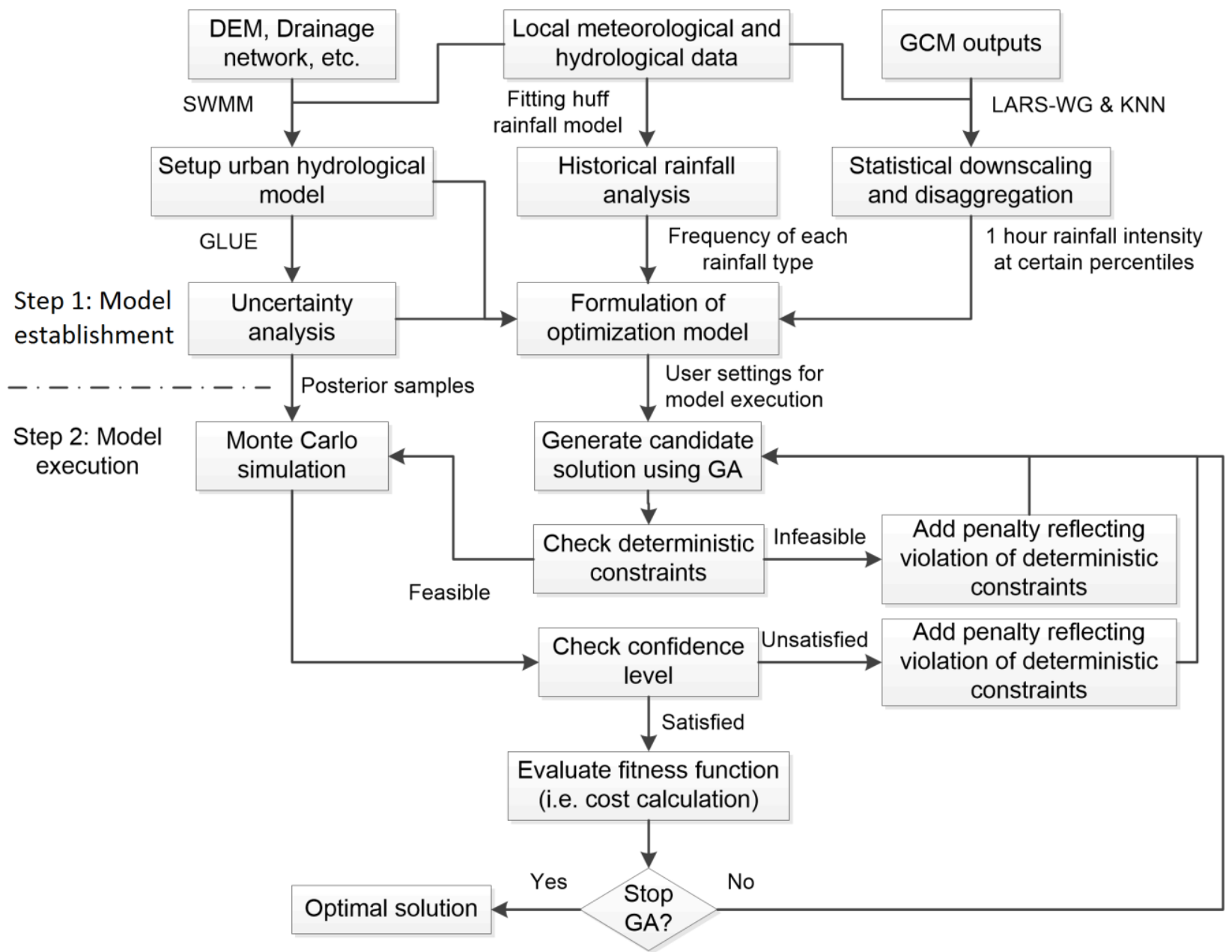

Place Figure 1 here

119

Step 1. Model establishment

123 Firstly, the required data of the study area need to be collected. They include: (i) the elevations 
129 calibrated using the observations (e.g. water depth and rainfall) of selected storm events. In this 130 study, the EPA SWMM is employed. Other urban hydrological models like MOUSE and 131 Infoworks are also applicable after proper calibrations. The generalized likelihood uncertainty 132 estimation (GLUE) (Beven and Binley, 1992) method is applied to derive the posterior 133 distribution of uncertain parameters (e.g. roughness coefficients) (see Section 2.2). Thirdly, the 134 historical rainfall record is analyzed to fit a suitable rainfall design model (e.g. Huff) to 135 determine the proportions of various rainfall types. It is used together with GCM outputs by 136 statistical downscaling to project future extreme rainfall conditions. The variability of the future 137 rainfall is determined based on ensembles of GCM outputs under various emission scenarios (see 138 Section 2.3). Finally, a stochastic optimization model for urban drainage design is formulated to 139 seek cost-effective solutions, where the hydrological simulation model is used to provide flood 140 information for checking satisfaction of constraints (see Section 2.4).

Step 2. Model execution

143 In order to run the model, users are required to customize some parameters of the model which 144 include: (i) the drainage design scenarios (e.g. nomination of conduits for optimization); (ii) the 145 acceptable flood damage (e.g. surcharge volume); (iii) the expected probability of non-flooding; 146 (iv) future extreme rainfall considering climate change effect. The GA is employed to solve the 147 stochastic optimization model formulated in Step 1. It is a directional search technique based on 148 a series of operations (e.g. initiation, evaluation, selection, crossover and mutation) analogous to 149 evolutionary biology (Homaifir et al., 1994). In applying GA, the potential solution is first 150 checked by using deterministic constraints to meet the hydraulic principles (i.e. ensure a 151 successful run of SWMM model) and regional limitations. The Monte Carlo (MC) simulation is 
152 then used to run the urban hydrological model repeatedly using the posterior uncertain

153 parameters as inputs. The flooding scenarios are extracted from the simulated results and the

154 probability of non-flooding is calculated. A penalty (i.e. a large value) will be added to the cost

155 to reflect the violation of deterministic or stochastic constraints and to ensure the non-optimal

156 potential solutions be rejected in the GA searching process. The optimal solution is obtained

157 finally when the stop criteria are met (e.g. a pre-defined number of generations or time limit is 158 reached).

\subsection{Urban Hydrological Modeling and Uncertainty Analysis}

160

161

162

163

$$
L(\boldsymbol{\theta})=\prod_{i=1, j=1}^{n, m} \exp \left[\frac{-\left(d_{i, j}^{m}-d_{i, j}^{o}\right)^{2}}{d_{i, j}^{o^{2}}}\right]
$$

173 where $\boldsymbol{\theta}$ denotes the vector of uncertain parameters; $d_{i, j}^{m}$ and $d_{i, j}^{o}$ are the simulated and 
174 observed water levels at the $i^{\text {th }}$ site under the $j^{\text {th }}$ storm event; $n$ is the number of observation sites;

$175 m$ is the number of storm events in calibration.

\subsection{Climate Change Scenarios and Rainfall Analysis}

The intensity-duration-frequency (IDF) curves are used to describe typical characteristics of the rainfall extremes. They are developed from frequency analysis on annual maximum rainfalls with different durations and normally used in urban drainage design (Arnbjerg-Nielsen et al., 2012). To address climate change impacts, the study results from Lu et al. (2015) are used. In this work, a combined statistical downscaling and disaggregation framework was applied to link the GCM outputs with local observations and generate rainfall time series in an hourly resolution. The hourly IDF curve was projected for future conditions, which was presented in intervals considering multiple ensembles (namely, 12 climate change scenarios obtained from four GCMs, i.e. CCSM3, HadCM3, IPCM4, and ECHAM5, with three emission scenarios, i.e. A1B, A2 and B1). This intends to address the intrinsic uncertainties in both climate models and different levels (e.g. high, median and low) of anthropogenic emissions. The selected scenarios have been extensively studied by many previous research works (Dawadi and Ahmad, 2012; Zhang et al., 2015). The maximum value of the projected 10-year hourly IDF curve (i.e. $98 \mathrm{~mm} / \mathrm{hr}$ ) in 2050s is adopted in this study to represent the future extreme rainfall condition ( $\mathrm{Lu}$ et al., 2015). Technical details of the methodology and results can be found in Lu et al. (2015).

In addition, the distribution of extreme rainfall (i.e. hyetograph) is also an influencing factor to the simulated flow outputs and the effectiveness of drainage design. This study assumes that the hourly rainfall in the study area follows the Huff storm distribution (Chow et al., 1988; Le et al., 2014), which includes four types of rainfall distributions. Le (2014) used the historical rainfall 
events with durations close to 1 hour in a similar location to fit the Huff rainfall distributions and his results showed that the relative proportions of rainfall distributions following Huff Type I, II, III and IV are $17.3 \%, 51.1 \%, 14.9 \%$ and $16.7 \%$, respectively. These values are adopted in the optimization model of drainage design to calculate the probability of flooding considering the uncertainty associated with the rainfall patterns. Detailed discussion on rainfall analysis can be found in Le (2014).

\subsection{Formulation of the Stochastic Optimization Model}

The problem under consideration is to seek optimal solutions for urban drainage sizing (i.e. to determine the engineering parameters or specifications for drains) in order to minimize the construction cost and at the same time satisfy the hydraulics constraints and flood mitigation criteria. Considering the uncertainty from urban hydrological simulation as well as the impact of climate change on designed rainfall, the optimal solution could help achieve the flood mitigation goal with some allowable risks. A chance-constrained programming $(\mathrm{CCP})$ model can be formulated to tackle such a problem. A generic CCP model is written as (Huang, 1998):

$$
\operatorname{Min} f(\mathbf{x}, \boldsymbol{\xi})
$$

subject to

$$
g_{i}(\mathbf{x}) \leq 0, i=1,2, \ldots, m
$$

$$
\operatorname{Pr}\left\{h_{j}(\mathbf{x}, \xi) \leq 0 \mid \xi\right\} \geq \alpha_{j}, j=1,2, \ldots, n
$$

where $f$ is objective function with decision variables $\mathbf{x}$ and random parameters $\xi ; g$ and $h$ denote the constraint functions with and without random parameters, respectively; $i$ and $j$ are the indexes of deterministic and uncertain constraints, respectively; $m$ and $n$ are the total numbers of $i$ and $j$, respectively; $\alpha$ is the probability of the corresponding uncertain constraint being satisfied. Specifically, the CCP model for urban drainage sizing subjected to uncertainty could be 
formulated as follows (Qin et al., 2010; Yu et al., 2015a):

221

$$
\operatorname{Min} C=a \sum_{i=1}^{n} V_{i}+b \approx c \sum_{i=1}^{n}\left(w_{i} \times d_{i}-w_{i}^{o} \times d_{i}^{o}\right) \times l_{i}^{o}+b
$$

222

223

224

225

226

227

228

229

230

231

232

233

234

235

236

237

238

239

240

241

subject to

$$
\operatorname{Pr}\{Q=S W M M(\mathbf{w}, \mathbf{d}, \xi, r) \leq \gamma \mid \xi, r\} \geq \alpha
$$

where $C$ is the construction cost of drainage sizing work, which is assumed to include excavation cost and management cost; $i$ is the index of the conduit $(i=1,2, \ldots, n) ; n$ is the total number of conduits; $a$ is the unit cost of evacuation including the cost related to excavation, transportation, disposal and labor for each cubic meter of excavation volume; $b$ is the management cost for operating drainage sizing project, which could be the cost indirectly linked to excavation work; $V_{i}$ is the excavation volume of the $i^{\text {th }}$ conduit under sizing consideration and estimated by the difference of drainage capacities between after and before sizing under the assumption that the cross section of the conduit is rectangular (as is the case in this study); $w_{i}^{o}, d_{i}^{o}$, and $l_{i}^{o}$ are the original width, depth and length of the $i^{\text {th }}$ conduit, respectively, where the length of the conduit is considered unchanged; $\mathbf{w}$ and $\mathbf{d}$ are the vectors of increased width and depth of drainage sizing, respectively; $\boldsymbol{\delta}$ and $\boldsymbol{\varphi}$ are the upper boundaries of $\mathbf{w}$ and $\mathbf{d}$, respectively; $Q$ is the total surcharging volume; $r$ is designed rainfall; $\alpha$ is the allowable probability of stochastic constraint satisfaction; $\gamma$ is acceptable threshold of flooding; SWMM denotes the simulation model driven by the designed rainfall ( $r$ ) with the inputs of conduit sizing parameters (i.e. $\mathbf{w}$ and $\mathbf{d}$ ); $\boldsymbol{\xi}$ is the 
242 matrix of stochastic parameters (e.g. roughness coefficients) representing the uncertainty

243 associated with the hydrological simulation model; $v_{j}$ is the surcharge volume at the $j^{\text {th }}$ junction;

$244 \omega$ is the threshold level of $v_{j}$; $t_{p}$ is the time that the flow takes to reach the peak flow at the outlet;

$245 \tau$ is the maximum allowable time for $t_{p}$.

247 Eq. 3a is the objective function which attempts to minimize the investment cost in consideration 248 of various drainage sizing options. In this study, the cost is assumed linearly proportional to the 249 total excavation volume for demonstrative purposes. Practically, the objective function should 250 consider more influencing factors such as conduit shapes, used materials and local economics. 251 The $\mathbf{w}$ and $\mathbf{d}$ are vectors of decision variables (i.e. design parameters of drainage sizing). The 252 dimension of $\mathbf{w}$ and $\mathbf{d}$ is equal to the number of conduits under consideration. Constraint $3 \mathrm{~b}$ is 253 defined to limit the total surcharge volume at all junctions to be less than an acceptable value 254 with a certain level of risk. $Q$ could be extracted from the simulation results of SWMM.

255 Constraint $3 \mathrm{c}$ allows a flexible consideration of protecting high priority areas (e.g. military and 256 historical site), whereas the overall flood-control target could still be achieved. Constraint $3 \mathrm{~d}$ is 257 used to control the time of reaching peak flow at the catchment outlet to meet an acceptable 258 criterion so that the impact of the rehabilitated drainage system on the downstream drains could 259 be minimized. Constraints $3 \mathrm{e}$ and $3 \mathrm{f}$ are technical constraints used to limit the candidate sizing 260 parameters vary within their possible ranges.

262 The probability of satisfying the stochastic constraint (the left side item of Eq. 3b) could be 263 estimated as:

$$
\operatorname{Pr}\{\cdot\}=\sum_{k=1}^{4} P_{k} \frac{N_{k}}{N_{t}}
$$


265 where $P_{k}$ is the relative proportion of the $k^{\text {th }}(k=1,2,3$ or 4$)$ Huff rainfall type based on

266 historical rainfall analysis; $N_{t}$ is the number of total simulations which is equivalent to the

267 number of posterior samples from GLUE analysis; $N_{k}$ is the number of simulations driven by the

$268 k^{\text {th }}$ type of Huff rainfall which lead to satisfaction of flood mitigation goals.

269

3. CASE STUDY

270

\subsection{Case Background and Model Setup}

271 A small drainage system in a typical urban catchment of Singapore is selected to demonstrate the

272 proposed SODD model. Some preliminary studies in the same catchment have been recently

273 reported by Yu et al. (2015a). Figure 2a shows the general map of the study site, which has a

274 catchment area of 32 hectares with elevations ranging from 105 to $130 \mathrm{~m}$ above the sea level. It

275 has a typically tropical climate, with abundant rainfall, high and uniform temperatures and high

276 humidity all year round. The average rainy days per year is 178 days and the long-term mean

277 annual rainfall is $2,331 \mathrm{~mm}$ with a typical duration around 1 hour (Meteorological Service

278 Singapore, 2015). The study site is mainly characterized by urban residential areas, parks and

279 roads. 


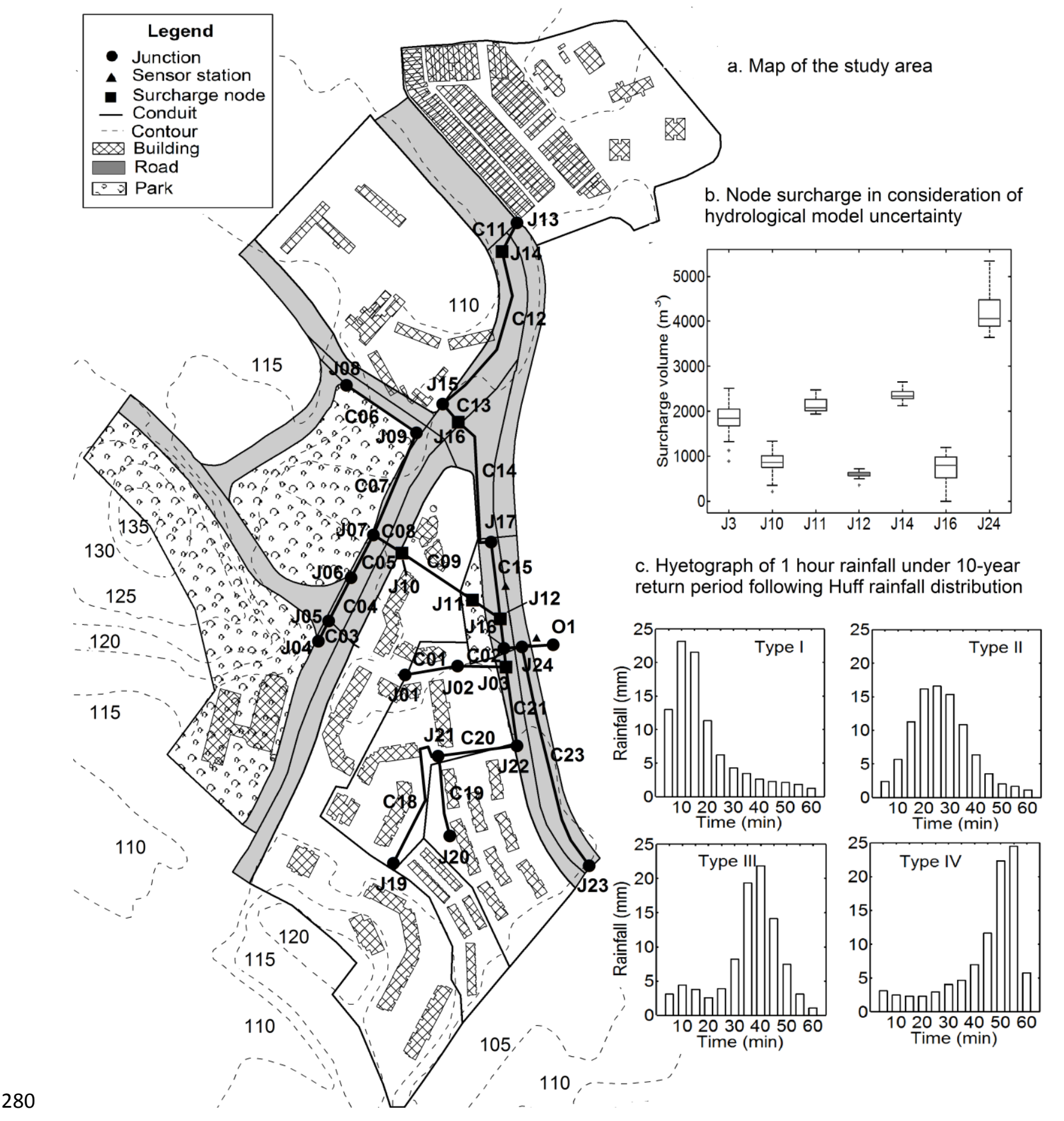

Place Figure 2

An urban hydrological model based on SWMM is setup to simulate the drainage system in this study case. The rains that fall in the catchment (i.e. 25 sub-catchments) are collected and routed 
to outfall O1 through 24 conduits. The cross-section type of the conduits is either open or closed rectangle. Based on the sensitivity test, the characteristic width of overland flow path (w) (i.e. a coefficient affecting the time of concentration in the conceptualized sub-catchment) and the roughness coefficients of impervious catchment area $\left(n_{i}\right)$, pervious catchment area $\left(n_{p}\right)$ and conduits $\left(n_{c}\right)$ are determined to be the most sensitive parameters in SWAT that affect the reliability of the simulated urban flows (Rossman, 2010). Considering the fact that $w$ varies in different sub-catchments, we propose to use the relative percentage change of $w$ to the default value estimated by SWMM as one of the uncertain parameters (see Supplementary Materials). A total of 5,000 simulations are executed with parameter sets sampled from prior distributions, where 628 parameter sets with the corresponding likelihood values (i.e. calculated based on Eq. 1) being larger than 0.95 are accepted as posterior samples. These posterior samples are used in MC simulations to estimate the probability (based on Eq. 4) of constraint satisfaction. The detailed description of SWMM model calibration and uncertainty analysis is provided in the Supplementary Materials. The most likely parameter set (e.g. leads to the maximum $L$ of 0.99 ) for $\left(w, n_{i}, n_{p}, n_{c}\right)$ is found to be $(-0.15 \%, 0.0097,0.1003,0.0166)$.

\section{Without losing generality, we select one of the typical drainage rehabilitation scenarios for} analysis, with the aim to optimize the size parameters of the studied drainage network to mitigate the flood risk under the storm event with a 10-year return period. According to the current local IDF curve (PUB, 2011), the peak intensity of 1-hour design storm is $91 \mathrm{~mm} / \mathrm{hr}$. Figure 2c shows the corresponding hyetographs of the disaggregated 1-hour design storm at a 10-year return period following four types of Huff rainfall distributions. The result shows that the existing drainage network could not accommodate excessive rainfall and would suffer from surcharging 
at nodes $\mathrm{J} 03, \mathrm{~J} 10, \mathrm{~J} 11, \mathrm{~J} 12, \mathrm{~J} 14, \mathrm{~J} 16$ and $\mathrm{J} 24$. Figure $2 \mathrm{~b}$ shows the variation of the surcharge volume at these nodes considering the parameter uncertainty of the hydrological model. The nodes of J03 and J24 that are near the outlet appear to have larger overflows (i.e., ranging from 1,200 to $2,600 \mathrm{~m}^{3}$ at $\mathrm{J} 03$ and from 3,800 to $5,300 \mathrm{~m}^{3}$ at $\mathrm{J} 24$, respectively). The overflows at J11, $\mathrm{J} 12$ and $\mathrm{J} 14$ fluctuate around 2,000, 600 and 2,200 $\mathrm{m}^{3}$, respectively, which are relatively less sensitive to model uncertainties. In addition, the future climate change could increase the extreme rainfall in the study area. According to Lu et al. (2015), the projected maximum value of 10-year hourly rainfall in consideration of future climate change (in 2050s) could increase about $7.7 \%$ (from 91 to $98 \mathrm{~mm} / \mathrm{hr}$ ). It will apparently result in even worse flash flood condition and thus should be considered in drainage network designs.

In total, two stochastic optimization models for urban drainage sizing are formulated under the present and future extreme rainfall scenarios. For the convenience of methodology demonstration, the optimization model (i.e. Eq. 3) is simplified with the following settings: (i) the unit cost $(a)$ is assumed to be $\mathrm{S} \$ 500$ per $\mathrm{m}^{3}$ to address the cost of evacuation, material, disposal and labor based on consultation with local contractors; (ii) the management cost $(b)$ is assumed to be zero for simplicity as it may vary with the scale of the contractor; (iii) the acceptable total surcharge volume $(\gamma)$ is set at $1,000 \mathrm{~m}^{3}$; (iv) the maximum expansion width $(\delta)$ and depth $(\varphi)$ for each conduit (i.e. decision variables) are limited to $3 \mathrm{~m}$ and $0.5 \mathrm{~m}$, respectively; (v) the time to peak flow $(\tau)$ at outfall $\mathrm{O} 1$ is less than 30 minutes (to keep it the same as that before drainage sizing); (vi) the probability of constraint satisfaction $(\alpha)$ is selected from 0.75 to 0.95 at an increment of 0.05 for comparison purposes. 
It should be noted that all conduits in this study are under sizing consideration and the constraint for individual nodes (i.e. Eq. 3c) is not applied. This is to simplify the optimization model and save the computational resources for demonstration. In practical applications, the conduits under sizing consideration and the corresponding constraints should be carefully examined based on local requirements. Furthermore, deterministic optimization models under present and future scenarios are also established for comparison purposes. The formulated optimization model was solved by GA in the Matlab environment, in which a $\mathrm{C}++$ based program was linked to "call" SWMM and extract the necessary information. The GA was set to run maximally 20 generations with a population size of 20 and a crossover fraction of 0.8 . It took about 2 hours to solve the deterministic optimization model and approximately 50 hours for the stochastic one in a PC equipped with Intel Core i5 3.4 GHz system and 8G RAM.

\subsection{Results and Discussions}

Figure 3 shows the simulated minimum costs from the deterministic optimization model under both present and future scenarios. Obviously, the investment cost would decrease with an increase of the total surcharge threshold (e.g. acceptable flood damage). The cost would decrease from 0.54 to 0.21 million $\mathrm{S} \$$ (at present) and from 0.8 to 0.49 million $\mathrm{S} \$$ (in the future), respectively, when the threshold of total surcharge volume increases from 500 to $2,000 \mathrm{~m}^{3}$. However, the marginal contribution of the investment (i.e. effect of flood control with the same investment) becomes low gradually. Under the present scenario, the investment would almost double (increase from 0.3 to 0.54 million $\mathrm{S} \$$ ) when the surcharge threshold decreases from 1,000 to $500 \mathrm{~m}^{3}$; whereas, the investment only increases slightly (from 0.27 to 0.3 million $\mathrm{S} \$$ ) when the threshold decreases from 1,500 to $1,000 \mathrm{~m}^{3}$. The results also indicate that the investment would need to increase by $146.7 \%$ (i.e. from 0.3 to 0.74 million $\mathrm{S} \$$ ) under the scenario of an allowable 
356 surcharge of $1,000 \mathrm{~m}^{3}$ considering the effect of future climate change (i.e. to cope with an

357 increase of hourly extreme rainfall by $7.7 \%$ ). It is noted that the deterministic solution could still

358 lead to flooding problems at a certain level of probability due to existence of the model

359 parameter uncertainty. As shown in Figure 3, the probability of being flooded (i.e. the total

360 surcharge exceeds the corresponding threshold) varies from $20 \%$ to $40 \%$ under various allowable

361 surcharges for both present and future conditions. Interestingly, a less stringent condition (i.e.

362 surcharge threshold of $2,000 \mathrm{~m}^{3}$ ) may lead to a quicker convergence of GA to yield the optimal

363 solution. This result would be more easily affected by uncertainty factors (e.g. model parameters)

364 and would lead to a higher flooding probability (e.g. 39\% in this study). It is also indicated that

365 the instability (e.g. random convergence) of GA solution in a parametric problem with respect to

366 the fitness function would increase the uncertainty of the overall system. Generally, the

367 deterministic optimization model is computationally efficient with no randomness being

368 considered in the hydrological simulation, whereas its solution is hardly reliable in achieving the 369 target of flood mitigation. 


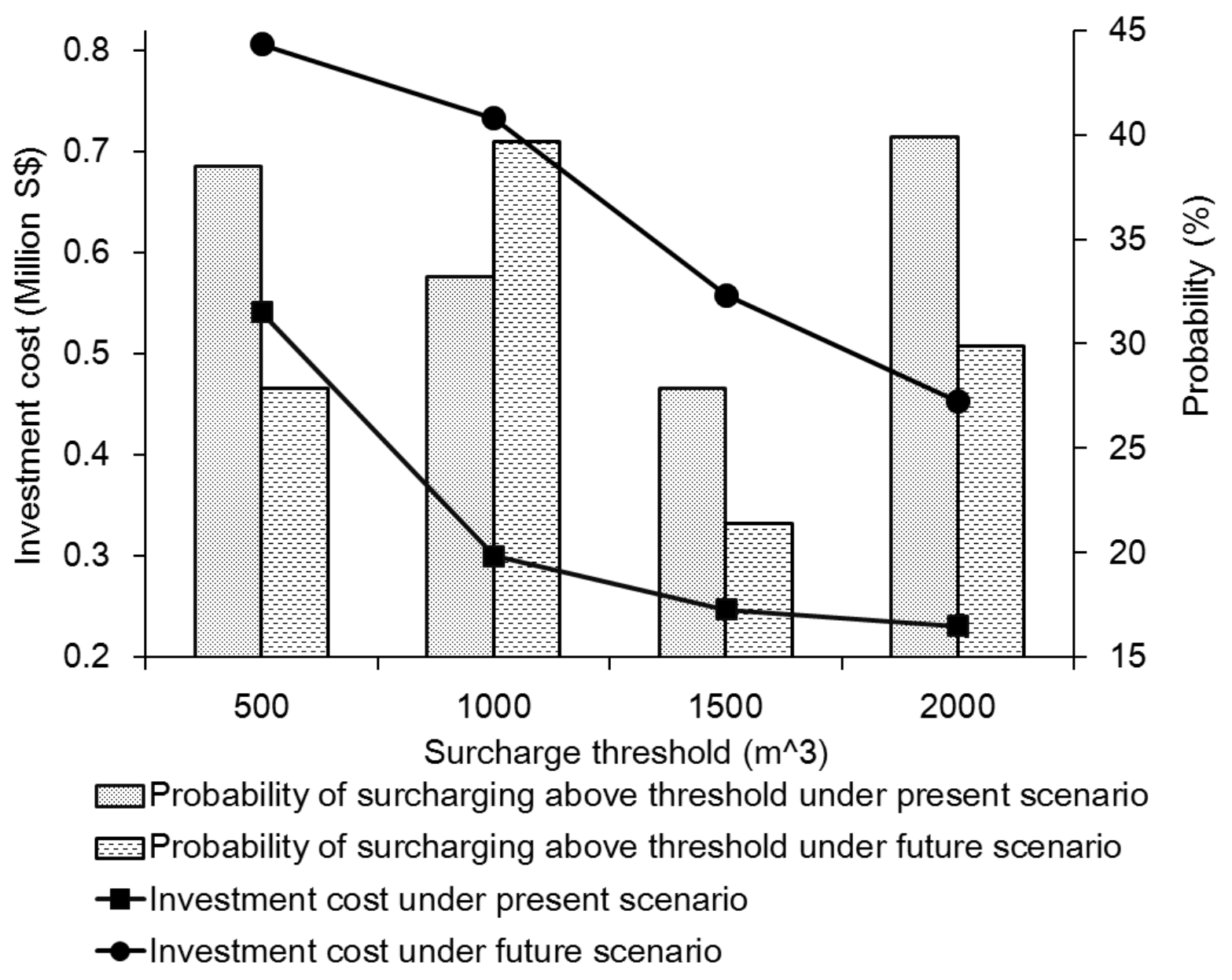

Place Figure 3 here

Figure 4 illustrates the minimum costs simulated from the stochastic optimization model under various probabilities of constraint satisfaction. The threshold of the total surcharge volume $(\gamma)$ is set to $1,000 \mathrm{~m}^{3}$. It is revealed that the investment would need to increase from 0.73 to 0.94 million $\mathrm{S} \$$ under the present scenario and from 1.23 to 1.52 million $\mathrm{S} \$$ under the future one, respectively, when the probability of constraint satisfaction increases from 0.75 to 0.95 . The investment would need to increase in average 1.65 times to accommodate the excessive rainfall

381 in the future in order to achieve a similar level of flood control as present. The amplitude is higher than that of improving the system reliability (i.e. to increase the probability of constraint 
383 satisfaction from 0.75 to 0.95 ), which is 1.29 times. It implies that the future climate change (i.e.

384 rainfall) has a relatively high impact on investment of drainage rehabilitation than the

385 hydrological model parameter uncertainty. Consequently, the solution under the present scenario

386 would unavoidably lead to an increased risk of flooding under changing climatic conditions in

387 the future. The probability of suffering from flooding varies from around $30 \%$ to $50 \%$ under

388 different optimization scenarios (see the gray bars in Figure 4). The results also show that, in

389 order to achieve a high level of system reliability (e.g. the probability of constraint satisfaction is

390 at a level of 0.9 ), the cost would be approximately 2.87 times higher than that from deterministic

391 model. Furthermore, the required cost would also increase notably to about 2.04 times higher if

392 the climate change effect is taken into consideration. Compared with the deterministic

393 optimization, the stochastic one could offer richer information on system reliability to facilitate a

394 risk-based decision making. Also, both flood risks and climate change effects could be

395 considered by the proposed model. 


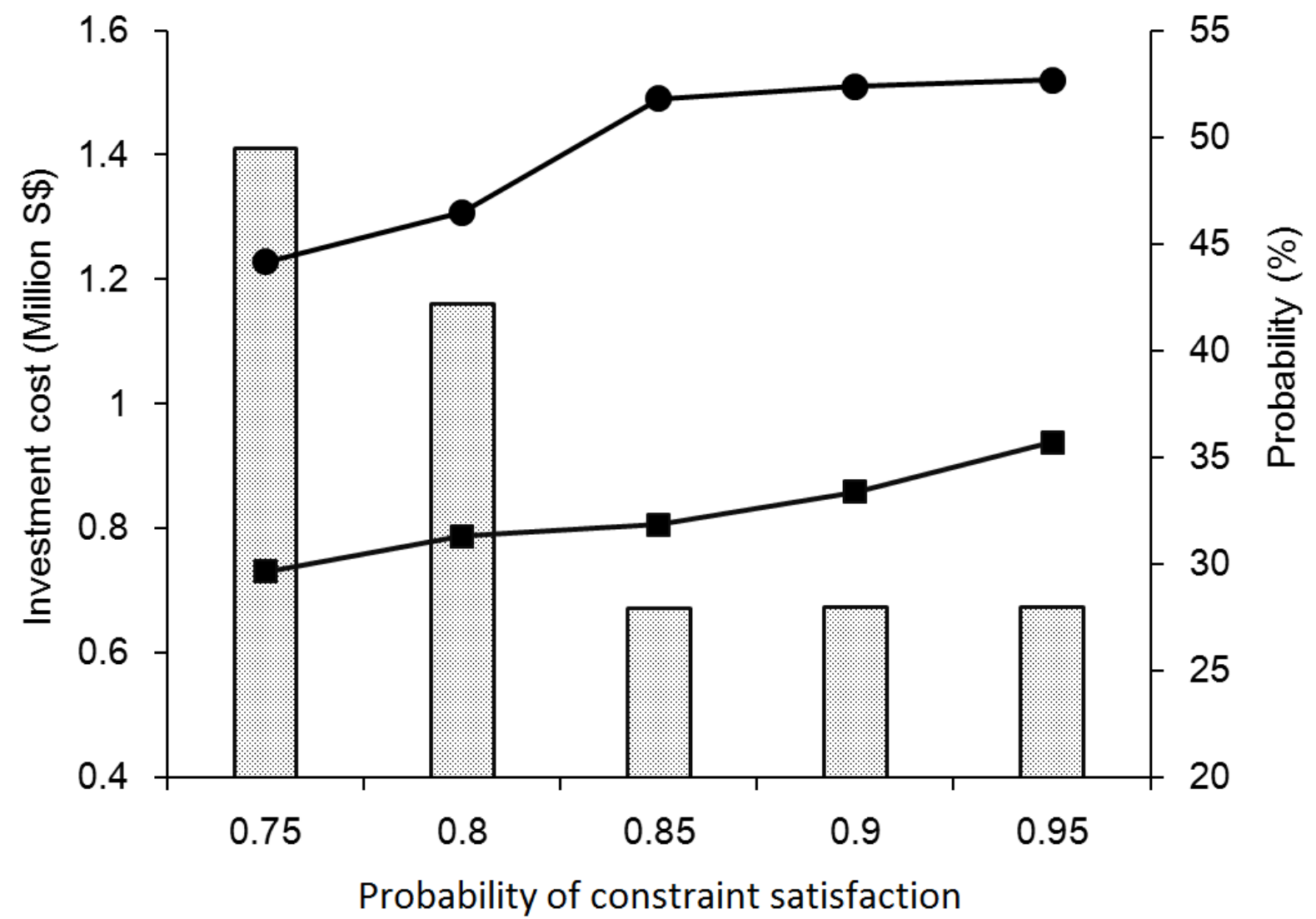

Probability of surcharging above threshold considering future scenario - -Investment cost under present scenario

$\rightarrow$ Investment cost under future scenario

Place Figure 4 here

399

Figure 5 shows the optimal solutions (i.e. the drainage size of the 24 conduits required for widening and digging) obtained from the deterministic and stochastic optimization models under both present and future scenarios. Generally, the solution from the deterministic model is relatively more economical and requires an average digging level of $0.13 \mathrm{~m}$ and a widening level 404 of $0.19 \mathrm{~m}$. Applying the stochastic optimization model for improving system reliability requires 405 a similar level of digging (i.e. averagely $0.22 \mathrm{~m}$ ) and a slightly lower level of widening (i.e. 
reduced from $0.8 \mathrm{~m}$ to $0.6 \mathrm{~m}$ in average) than those considering the effect of increased rainfall extremes under climate change. When both factors are taken into consideration, the drainage capacity would need significant improvement compared with the solution from the deterministic optimization. The average levels of digging and widening are $0.22 \mathrm{~m}$ (from 0.15 to $0.5 \mathrm{~m}$ ) and $1.53 \mathrm{~m}$ (from 0.8 to $2.8 \mathrm{~m}$ ), respectively.

Figures $6 \mathrm{a}$ and $6 \mathrm{~b}$ demonstrate the flooding scenarios (i.e. surcharge nodes) in a conceptualized drainage layout (from Figure 2a) in applying the optimal solutions (as shown in Figure 5) under various scenarios. The surcharge is due to the allowable flood damage (i.e. threshold) set in the optimization models. It is indicated that the solution from the deterministic model would lead to a more extensive flooded areas (e.g. 5 surcharge nodes); and that from the stochastic one would have surcharge problems at one or two nodes. This suggests that the stochastic model is more robust to the effect of uncertainty associated with the urban hydrological model which may result in a spatial variation of flooding. Figures $6 \mathrm{~b}$ to $6 \mathrm{e}$ show the variation of surcharge volume at individual nodes, which could aid decision makers understand the extreme flood risks. For example, at extreme conditions shown in Figures $6 \mathrm{~d}$ and $6 \mathrm{e}$, the volumes of surcharges under the present condition would increase to around 1,500 and $1,000 \mathrm{~m}^{3}$ for $\mathrm{J} 10$ and $\mathrm{J} 16$, respectively; those under the future condition would increase to around $1,300 \mathrm{~m}^{3}$ for $\mathrm{J} 24$. If necessary, post-optimization analysis could be used to help understand the actual effect in adopting the optimal solution and explore better optimization results by changing local settings (e.g. installment of storm water detention tanks). 

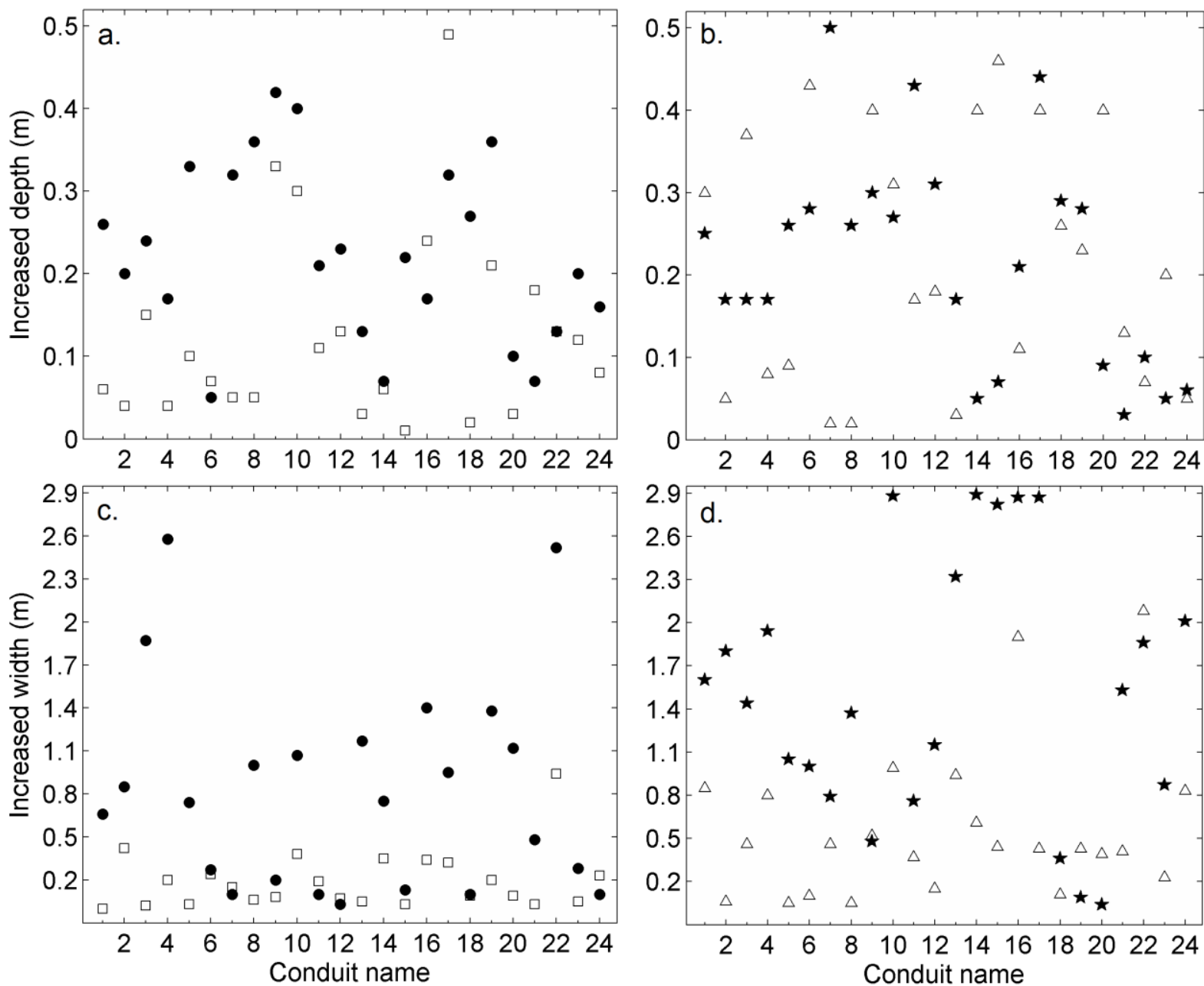

- Solution of deterministic optimization (present)

$\triangle$ Solution of deterministic optimization (future)

- Solution of stochastic optimization (present)

$\star$ Solution of stochastic optimization (future)

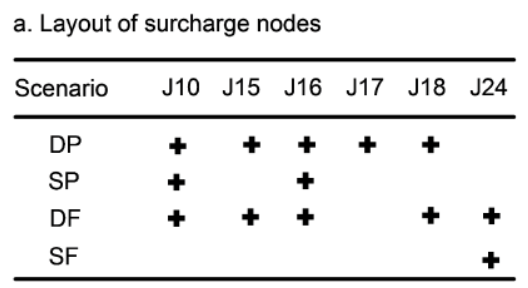

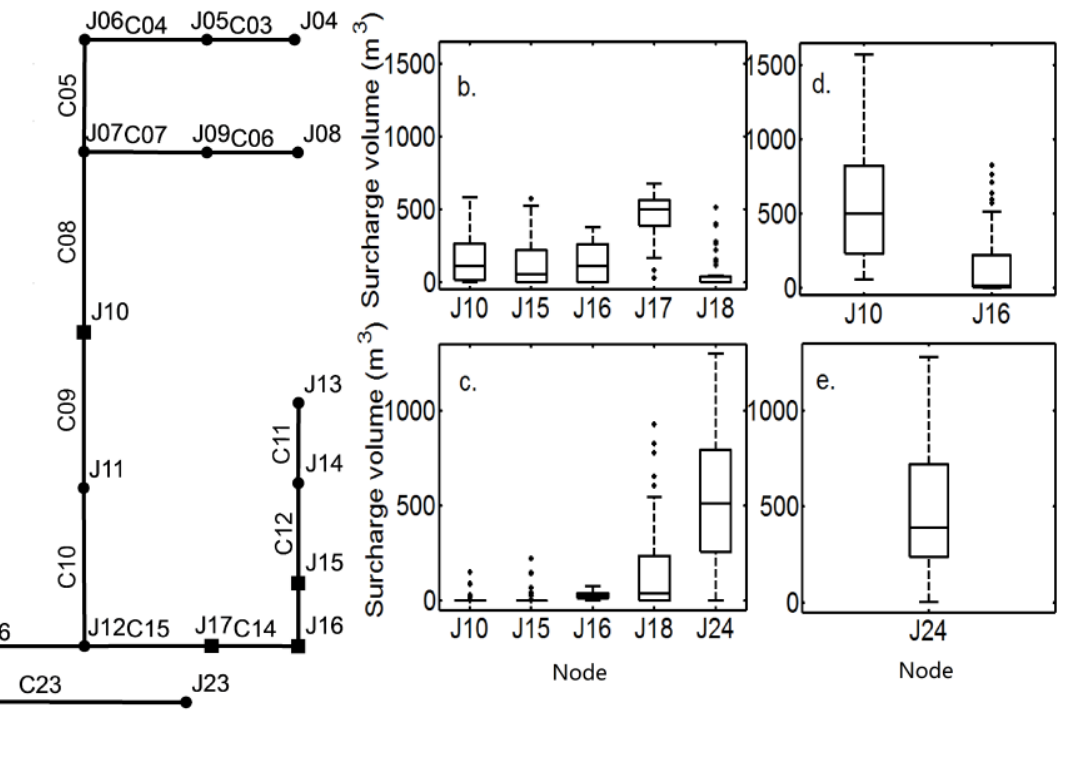


Place Figures 5 and 6 here

Figure 7 shows the comparison of the simulated minimum cost (for the present scenario) under various likelihood thresholds ( $L$ in Eq. 1 ) selected for uncertainty analyses. The threshold is considered to affect the number of the accepted posterior samples and the system reliability (i.e. calculated probability of constraint satisfaction during stochastic optimization). Generally, the cost would increase with the decrease of the likelihood threshold (from 0.95 to 0.9 ) at the same probability of constraint satisfaction. This is because the decrease of likelihood threshold would result in an increased number of simulations which may involve additional flooding scenarios. Consequently, a relatively higher cost would be needed to ensure achievement of a pre-defined system reliability. However, the probability of constraint satisfaction seems to affect the yield solutions more notably than the selected likelihood threshold $(L)$. For example, the investment would increase around $30 \%$ to improve the probability of constraint satisfaction from 0.75 to 0.95 ; however, at a probability level of 0.85 (as an example), the investment would only increase by $7.5 \%$ if $L$ decreases from 0.95 to 0.9 . The effect of model parameter uncertainty would be insignificant when $L$ is set to 0.975 (corresponding to 365 posterior samples), implying that the cost is no longer sensitive to the probability of constraint satisfaction. This reveals that if the likelihood threshold is properly selected, the targeted probability of constraint satisfaction would cause more significant effect on the objective functions (i.e. investment cost). Such a conclusion may be specific in this study case and may not be general. Nonetheless, the analysis of the parameter effect could help understand the relative importance of sensitive factors. 


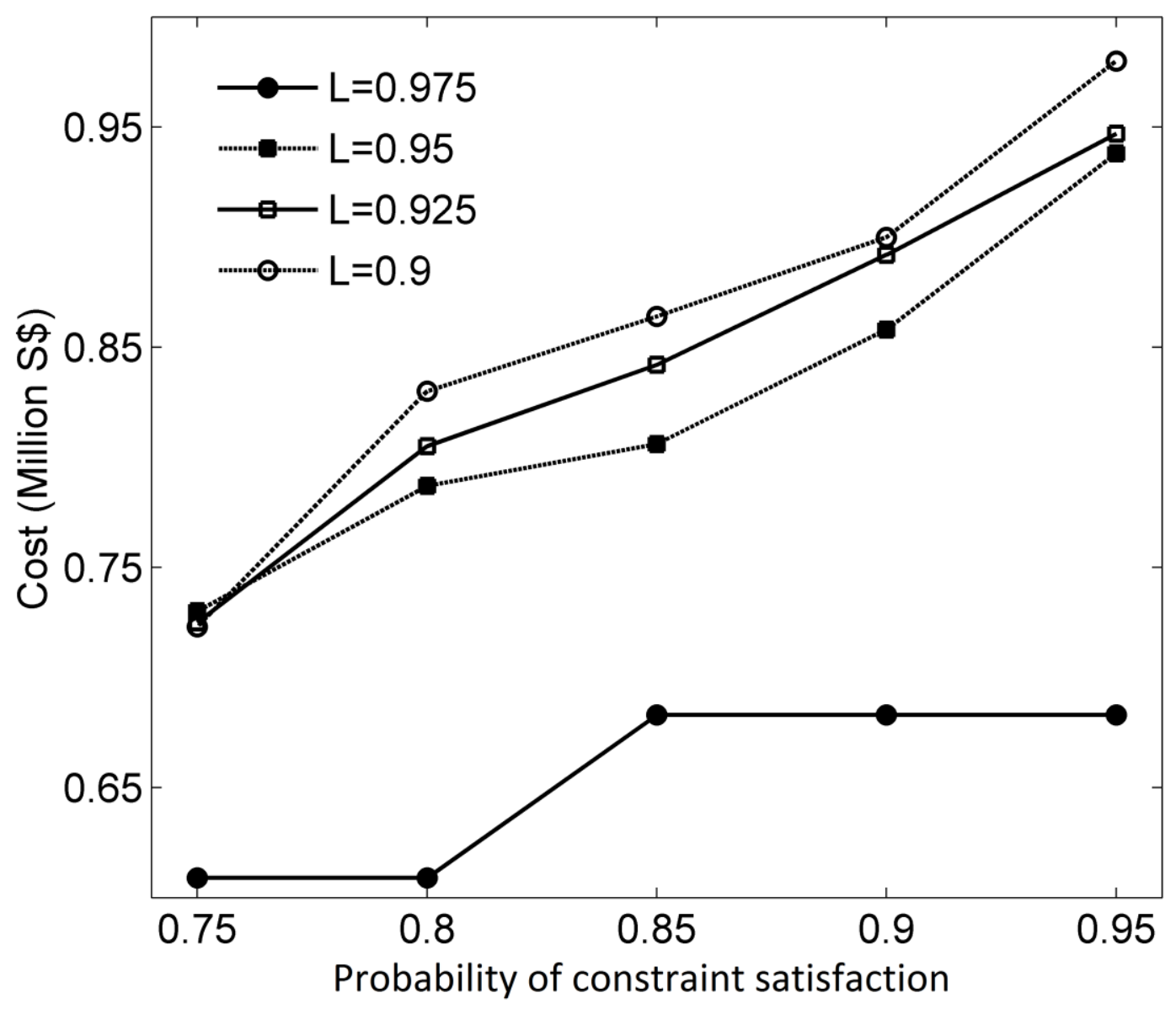

Place Figure 7 here

455

456

The proposed SODD model involves various techniques including urban hydrological modeling, climate model downscaling, uncertainty analysis, chance-constrained programming and heuristic algorithm to obtain optimal solution of drainage design under complexities. The model allows generation of robust optimal solutions which could be used by decision makers to conduct a tradeoff analysis between the risk of being flooded and the total investment. The model could 462 also help decision makers better understand the potential impact of climate change on the 463 drainage design and the additional cost arising from adaptation. More importantly, the SODD 464 model is flexible for various practical applications. For example, the urban hydrological model 
could be adapted to reflect specific conditions for other locations without changing the overall methodology framework. In addition, the optimization model could be easily modified for tackling other types of drainage design problems such as placement of storm water detention tanks (Tao et al, 2014; Yu et al., 2015b).

There are also a number of concerns in applying the proposed SODD model. First, this study considered all the conduits for optimization; whereas in practical applications, users could narrow down the considerations based on their empirical experience and local requirement. The integer programming could also be incorporated to reflect options of switching on or off a specific conduit for automatically judging the number of conduits for optimization. Second, the limitation of social economic data in this study area and the absence of a 2-dimensional inundation model to predict distributed water depths restricted monetizing the flood damages. Third, the drainage sizing optimization was conducted using designed rainfall. The climate change could affect the rainfall variability and, consequently, the optimal solution of drainage design. In this study, we evaluated the climate change impact on the drainage design investment considering the achievement of flood mitigation goal at present. But, there is a lack of sufficient consideration of antecedent conditions and evolution of the changing climate, which requires further exploration of adaptive decision-making strategies for a resilient drainage network planning. Finally, the computational requirement was intensive for solving a large-scale optimization problem involving both numerical modeling and MC-based stochastic simulations. For similar studies, it is advised to estimate the demand of computational resources first by referring to this study case to ensure the feasibility of application. The surrogate approaches (Yu et al., 2015c, Yazdi et al., 2016), which attempt to establish computationally efficient data-driven 
models to approximate the numerical simulations, may be promising approaches for further explorations. The possibility of applying cloud computing or parallel computing cost-effectively could also be a viable solution (Xu et al., 2015).

\section{CONCLUSIONS}

A stochastic optimization model for urban drainage design (SODD) was developed in this study. It integrated techniques of urban hydrological simulation, uncertainty analysis, climate change model downscaling and heuristic algorithm to solve a stochastic optimization model considering the tradeoff between investment of drainage rehabilitation and acceptable flood risks. A case study of drainage sizing problem in the tropical area was selected for demonstration. The results showed that although the solution from the deterministic optimization was economical, its application could lead to flooding due to parameter uncertainties. The results also showed that the future climate change had a significant impact on the simulated cost compared to that of the acceptable probability of constraint satisfaction. Compared with recent developments in the related area, the proposed SODD model is novel in the sense that (i) it has the advantage of dealing with uncertainty and addressing climate change simultaneously to enable a robust optimization for urban drainage design; (ii) it could be utilized by stakeholders to conduct cost-effective climate change adaption planning regarding urban drainage rehabilitation; and (iii) it has the potential to be tailored for various practical applications, e.g. design of storm water detention tanks and incorporation of best management practices. The limitations of expensive computational requirement and absence of inundation modeling are desired to be enhanced in our future studies.

\section{ACKNOWLEDGEMENTS}

The research work was supported by Singapore's Ministry of Education (MOE) AcRF Tier 1 
511 Project (Ref No. RG188/14; WBS No.: M4011420.030) and Nanyang Technological University

512 (NTU) Start-Up Grant (WBS No.: M4081327.030). The authors also deeply appreciate the

513 reviewers' comments, which have contributed much to improving the manuscript.

\section{REFERENCES}

515 Aad, M. P. A., Suidan, M. T., and Shuster, W. D. (2009). "Modeling techniques of best management practices: Rain barrels and rain gardens using EPA SWMM-5.” J. Hydrol. Eng., 10.1061/(ASCE)HE.1943-5584.0000136.

Arnbjerg-Nielsen, K. (2012). “Quantification of climate change effects on extreme precipitation used for high resolution hydrologic design.” Urban Water J., 9(2), 57-65.

Ashofteh, P., Haddad, O., and Loáiciga, H. (2015). "Evaluation of Climatic-Change Impacts on Multiobjective Reservoir Operation with Multiobjective Genetic Programming." J. Water Resour. Plann. Manage., 10.1061/(ASCE)WR.1943-5452.0000540, 04015030.

Beven, K. J., and Binley, A. (1992). "The future of distributed models: model calibration and uncertainty prediction.” Hydrol. Process., 6, 279-298.

Chow, V.T., Maidment, D. R., and Mays, L.W. (1988). Applied hydrology. McGraw-Hill Book Company, Maidenherd.

Cimorelli, L., Cozzolino, L., Covelli, C., Della Morte, R., and Pianese, D. (2014). "Enhancing the efficiency of the automatic design of rural drainage networks." J. Irrig. Drain Eng., 10.1061/(ASCE)IR.1943-4774.0000721, 04014015.

Cozzolino, L., Cimorelli, L., Covelli, C., Mucherino, C., and Pianese, D. (2015). “An innovative approach for drainage network sizing." Water, 7(2), 546-567.

Dawadi, S. and Ahmad, S. (2012). "Changing climatic conditions in the Colorado River Basin: Implications for water resources management”. J. Hydrol., 430-431, 127-141. 
Duan, H., Li, F. and Tao, T. (2016). "Multi-objective Optimal Design of Detention Tanks in the Urban Stormwater Drainage System: Uncertainty and Sensitivity Analysis”. Water Resour. Manage., doi:10.1007/s11269-016-1282-1.

Dutta, S., Sahoo, B., Mishra, R. and Acharya, S. (2016) "Fuzzy Stochastic Genetic Algorithm for Obtaining Optimum Crops Pattern and Water Balance in a Farm”, Water Resour Manage, doi:10.1007/s11269-016-1406-7.

Gironás, J., Roesner, L. A., Rossman, L. A., and Davis, J. (2010). “A new applications manual for the Storm Water Management Model (SWMM)." Environ. Modell. \& Softw., 25(6), 813-814.

Haghighi, A., and Bakhshipour, A. E. (2014). "Deterministic integrated optimization model for sewage collection networks using tabu search.” J. Water Resour. Plann. Manage., doi:10.1061/(ASCE)WR.1943-5452.0000435, 04014045.

Hammond, M. J., Chen, A. S., Djordjević, S., Butler, D., and Mark, O. (2015). “Urban flood impact assessment: A state-of-the-art review.” Urban Water J., 12(1), 14-29.

Homaifar, A., Qi, C. X., and Lai, S. H. (1994). “Constrained optimization via genetic algorithms.” Simulation, 62(4), 242-253.

Huang, G. H. (1998). “A hybrid inexact-stochastic water management model.” Eur. J. Oper. Res., 107(1), 137-158.

Jacobs, T., Medina, M., Jr., and Ho, J. (1997). "Chance Constrained Model for Storm-Water System Design and Rehabilitation." J. Water Resour. Plann. Manage., doi:10.1061/(ASCE)0733-9496(1997).

Li, F., Duan, H. F., Yan, H., and Tao, T. (2015). "Multi-objective optimal design of detention tanks in the urban stormwater drainage system: framework development and case study." 
Water Resour. Manag., 29(7), 2125-2137.

558

559

560

561

562

563

564

565

566

567

568

569

570

571

572

573

574

575

576

577

578

579

Le, Q.T. (2014). "Evaluation of urban drainage flow impacts under climate change using an integrated modeling framework." Qualification Exam Report submitted to Nanyang Technological University, Singapore, 2014.

Le, Q.T., Lu, Y., Qin, X.S., and Min, R. (2014). “Assessing climate change impact on urban drainage flow: A case study of Singapore." Proc. of the 13th International Conf. on Urban Drainage (ICUD2014), Sarawak, Malaysia, 1-9.

Lu, Y., Qin, X.S. and Mandapaka, P.V. (2015). “A combined weather generator and K-nearest-neighbour approach for assessing climate change impact on regional rainfall extremes.” Int. J. Climatol., 35(15), 4493-4508.

Lund, J. R. (2002). "Floodplain planning with risk-based optimization.” J. Water Resour. Plann. Manage., doi:10.1061/(ASCE)0733-9496.

Mailhot, A. and Duchesne, S. (2010). "Design Criteria of Urban Drainage Infrastructures under Climate Change.” J. Water Resour. Plann. Manage., 10.1061/(ASCE)WR.1943-5452.0000023.

Marchi, A., Dandy, G., and Maier, H. (2016). "Integrated Approach for Optimizing the Design of Aquifer Storage and Recovery Stormwater Harvesting Schemes Accounting for Externalities and Climate Change." J. Water Resour. Plann. Manage., 10.1061/(ASCE)WR.1943-5452.0000628, 04016002.

Meteorological Service Singapore. (2015). http://www.weather.gov.sg/climate-climate-of-singapore/, assessed on Jan. 14, 2016.

Muleta, M. K., and Boulos, P. F. (2007). "Multiobjective Optimization for Optimal Design of Urban Drainage Systems.” World Environmental and Water Resources Congress 2007, 1-10. Rossman, L.A. (2010). “Storm Water Management Model User's Manual version 5.0.” United 
States Environmental Protection Agency (EPA), EPA/600/R-05040.

581

Public Utility Board (PUB). (2011). "Code of Practice -7. Drainage Design and Considerations" http://www.pub.gov.sg/general/code/Pages/SurfaceDrainagePart2-7.aspx (Jan. 14, 2016)

Qin, X.S., Huang, G., and Liu, L. (2010). “A genetic-algorithm-aided stochastic optimization model for regional air quality management under uncertainty.” J. Air Waste Manage. Assoc., 60(1), 63-71.

Saraswat, C., Kumar, P., and Mishar B. K. (2016). “Assessment of stormwater runoff management practices and governance under climate change and urbanization: An analysis of Bangkok, Hanoi and Tokyo", Environ. Sci. Policy, 64, 101-117.

Sebti, A., Fuamba, M., and Bennis, S. (2015). "Optimization Model for BMP Selection and Placement in a Combined Sewer." J. Water Resour. Plann. Manage., 10.1061/(ASCE)WR.1943-5452.0000620, 04015068.

Sebti, A., Carvallo Aceves, M., Bennis, S., and Fuamba, M. (2016). "Improving Nonlinear Optimization Algorithms for BMP Implementation in a Combined Sewer System." J. Water Resour. Plann. Manage., 10.1061/(ASCE)WR.1943-5452.0000669, 04016030.

Tao, T., Wang, J., Xin, K., and Li, S. (2014). “Multi-objective optimal layout of distributed storm-water detention.” Int. J. Environ. Sci. Tech., 11(5), 1473-1480.

Thorndahl, S., Beven, K. J., Jensen, J. B., and Schaarup-Jensen, K. (2008). "Event based uncertainty assessment in urban drainage modelling, applying the GLUE methodology." $J$. Hydrol., 357(3), 421-437.

Thorndahl, S., and Willems, P. (2008). "Probabilistic modelling of overflow, surcharge and flooding in urban drainage using the first-order reliability method and parameterization of local rain series.” Water Res., 42(1), 455-466. 
Yazdi, J., Lee, E. H., and Kim, J. H. (2014). "Stochastic multiobjective optimization model for urban drainage network rehabilitation." J. Water Resour. Plann. Manage., 10.1061/(ASCE)WR.1943-5452.0000491, 04014091.

Yazdi, J., Torshizi, A.D., and Zahraie, B. (2016). "Risk based optimal design of detention dams considering uncertain inflows." Stoch. Environ. Res. Risk Assess., 30:1457-1471

$\mathrm{Xu}$, J, Huang, E, Chen, C H, and Lee, L H (2015). “Simulation optimization: a review and exploration in the new era of cloud computing and big data." Asia Pac. J. Oper. Res., 32(03), 1550019.

Yu, J.J., Qin, X.S., and Min, R. (2015a). “Optimization-Model-Aided Urban Drainage Upgrading Using Genetic Algorithm." Proc. of the 10th International Urban Drainage Modelling Conf. (UDM 2015), Mont-Sainte-Anne, Québec, Canada., pp.143-148.

Yu, J.J., Qin, X.S., and Min, R. (2015b). “Storm Water Detention Tank Design Considering Climate Change.” International Expert Workshop Towards Urban Water Security in Southeast Asia: Managing Risk of Extreme Events, Phnom Penh, Cambodia.

Yu, J.J., Qin, X.S, and Larsen, O. (2015c). "Uncertainty analysis of flood inundation modeling using GLUE with surrogate models in stochastic sampling." Hydrol. Process., 29(6), 1267-1279.

Zhang, Y., Su, F. Hao, Z., Xu, C., Yu Z., Wang, L., and Tong, K. (2015). “Impact of projected climate change on the hydrology in the headwaters of the Yellow River basin". Hydrol. Process., 29(20), 4379-4397.

Zhou, Q., Panduro, T.E., Thorsen, B.J., and Karsten A.N. (2013). “Adaption to Extreme Rainfall with Open Urban Drainage System: An Integrated Hydrological Cost-Benefit Analysis”. Environ. Manage. doi:10.1007/s00267-012-0010-8. 


\section{List of Figure Captions}

627 Fig. 1. The workflow of SODD.

628

629 Fig. 2. (a) The map of the study area, (b) node surcharge in consideration of urban hydrological

630 model parameter uncertainty, and (c) hyetograph of 1 hour rainfall under 10-year return period

631 following four types of Huff rainfall distributions.

632

633 Fig. 3. Simulated minimum cost from deterministic optimization and the corresponding

634 probability of being flooded in consideration of urban hydrological model parameter uncertainty. 635

636 Fig. 4. Simulated minimum cost from stochastic optimization under various probability of 637 constraint satisfaction.

638

639 Fig. 5. Yield solutions of drainage size required for digging (a) and widening (b) from 640 deterministic and stochastic optimization under both present and future scenarios.

642 Fig. 6. Post-optimization analysis of flooding scenarios: (a) layout of surcharge nodes (black

643 squares) in applying deterministic and stochastic optimizations; (b) and (c) are boxplots of node 644 surcharge volume in applying deterministic optimization under present and future scenarios, 645 respectively; (d) and (e) are boxplots of node surcharge volume in applying stochastic 646 optimization under present and future scenarios, respectively. Note: DP and DF denote 647 deterministic optimizations under present and future scenarios, respectively; SP and SF denote 648 stochastic optimizations under present and future scenarios, respectively; the black cross 649 indicates node surcharging under corresponding simulation scenarios. 650

651 Fig. 7. Simulated minimum cost under various probability of constraint satisfaction with 652 different likelihood thresholds in GLUE. 